NASA Technical Memorandum 101623

\title{
INTEGRATED STRUCTURE/CONTROL LAW DESIGN BY MULTILEVEL OPTIMIZATION
}

\author{
(NASA-TA-10 1623) INTEGRA TED \\ N89-26623 \\ STR UCTURE/CONT KOL LAN DESIGN BY MULTILEVEL \\ OPTIMIZATION (NASA. Langley Researcb \\ Center) $12 \mathrm{p}$ \\ CSCL O9B \\ $\begin{array}{ll}\text { Unclas } \\ \text { G3/63 } & 0222719\end{array}$
}

Michael G. Gilbert

David K. Schmidt

JUNE 1989

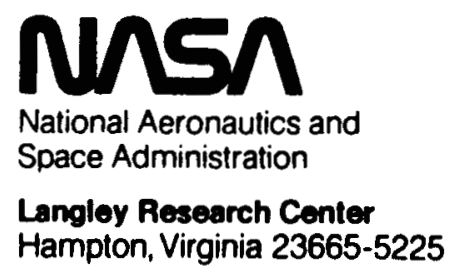




\title{
INTEGRATED STRUCTURE/CONTROL LAW DESIGN BY MULTILEVEL OPTIMIZATION
}

\author{
Michael G. Gilbert* \\ NASA Langley Research Center \\ Hampton, VA 23665 \\ David K. Schmidt ${ }^{\dagger}$ \\ Arizona State University \\ Tempe, AZ 85287
}

\begin{abstract}
A new approach to integrated structure/control law design based on multilevel optimization is presented. This new approach is applicable to aircraft and spacecraft and allows for the independent design of the structure and control law. Integration of the designs is achieved through use of an upper level coordination problem formulation within the multilevel optimization framework. The method requires the use of structure and control law design sensitivity information. A general multilevel structure/control law design problem formulation is given, and the use of LQG control law design and design sensitivity methods within the formulation is illustrated. Results of three simple integrated structure/control law design examples are presented. These results show the capability of structure and control law design tradeoffs to improve controlled system performance within the multilevel approach.
\end{abstract}

\section{Introduction}

Modern day air and space vehicles are complex systems made up of components which are often in and of themselves high-order dynamic systems. For example, the airframe, engine, and flight controls of a modern jet fighter aircraft individually require sophisticated mathematical modeling, simulation, and testing to achieve good performance with appropriate dynamic characteristics.

The integration of good individual dynamic components into a single aerospace vehicle can, however, result in dynamic interactions which lead to unsatisfactory

* Aerospace Engineer, Aeroservoelasticity Branch, Structural Dynamics Division. Senior Member AIAA.

† Professor, Department of Mechanical and Aerospace Engineering. Associate Fellow AIAA. vehicle performance. The F-16 and F-18 exhibited adverse dynamic interactions between the airframe aeroelastic characteristics and flight control systems in flight. The $X-29$ aircraft was predicted to have adverse dynamic interactions as well. These actual and predicted dynamics problems were ultimately fixed by expensive and time consuming flight control system redesigns. Similar dynamic interaction problems are predicted for such future large-scale space vehicles as the proposed space station and satellites with large solar arrays and antennas.

Analytical methods to predict aeroservoelastic stability of aircraft and servoelastic stability of spacecraft have been developed. The ability to predict servoelastic or aeroservoelastic interactions leads naturally to the desire to integrate, that is, include, dynamic interaction effects in aerodynamic, structure, and/or control system designs, such that adverse dynamic coupling in the final vehicle is eliminated. Numerous papers outlining integrated structure/control law design methods for large space structure applications have appeared in recent years. ${ }^{1-7}$

The purpose of this work is to develop the means to include dynamic vehicle response characteristics and well known control law design methods in an existing general framework for multidisciplinary (aerodynamics, propulsion, structures, etc.) design. The existing general framework is based on formal design problem decomposition, multilevel optimization techniques, and design sensitivity information. The formulation of a design problem decomposition for dynamic aerospace vehicles and the use of Linear Quadratic Gaussian (LQG) optimal control law design and sensitivity analysis methods leads to an alternative structure/control law design method which is applicable to both air and space vehicles. Development within the general framework retains the use of independent design tools for both the structure and the control law, and provides for the future addition of other design tools for aerodynamics, propulsion, and the like. 
The paper is organized as follows. A summary of existing structure/control law design methods and a summary of multilevel optimization theory are given first. These are followed by a description of the multilevel structure/control law design algorithm. Three structure/ control law design cases are presented for a twobar truss example problem. The first design case was to improve the closed-loop system stability robustness using control law and structural design freedoms, while the second and third cases were formulated to expressly examine structure and control law design tradeoffs.

\section{Existing Design Methods}

Numerous methods have been developed in recent years for structure/control law design of large space structure systems. These existing methods can generally be categorized by their approach to the problem as either simultaneous or sequential. ${ }^{1}$

Simultaneous Methods - A simultaneous structure/control law design method is one in which the control law design problem and the structural design problem are directly combined into a single problem. Examples of existing structure/control law design approaches which can be characterized as simultaneous include those reported by Haftka, Martinovic, and Hallauer ${ }^{2}$, Salama, Hamidi, and Demsetz ${ }^{3}, \mathrm{Hale}^{4}$, and Miller et. al. ${ }^{5}$

Sequential Methods - A sequential structure/control law design method is one in which the control law design and the structural design are conducted in an iterative, multiple step manner. Examples of reported sequential structure/control law design methods are those by Messac, Turner, and Soosaar ${ }^{6}$, and Khot et.al. ${ }^{7}$

\section{Multilevel Optimization Theory}

A theory of multilevel optimization for large scale engineering design problems has been developed in recent years. 8.9 This theory is based on hierarchal problem decomposition methods $8-11$ and the use of the sensitivity of optimization problem solutions to fixed problem parameters. ${ }^{12-14}$

The theory is perhaps best explained by use of a conceptual multilevel optimization problem as shown in Figure 1. The conceptual problem is to minimize the performance index $\mathrm{J}$ by selection of the design variable $\alpha$ at the upper level, and to minimize the performance indices $K$ and $L$ by selection of the design variables $\beta$ and $\chi$ respectively at the lower level. Note that in this example, $K$ and $L$ are functions of $\alpha$ and $\beta$ and $\alpha$ and $\chi$ respectively, while $\mathrm{J}$ is a function of $\alpha, \beta$, and $\chi$.
The basic idea behind multilevel optimization is to treat the design variable $\alpha$ as a fixed constant during the minimization of $K$ and $L$. With $\alpha$ fixed, the minimizations of $K$ and $L$ are decoupled, and are performed independently. Once the design variables $\beta=\beta^{*}$ and $\chi=$ $\chi^{*}$ which minimize $\mathrm{K}$ and $\mathrm{L}$ are known, the performance index $\mathrm{J}$ of the upper level minimization can be evaluated and the gradient of $J$ with respect to $\alpha$ computed.

Clearly the $\beta=\beta^{*}$ and $\chi=\chi^{*}$ which minimize $K$ and $\mathrm{L}$ respectively are functions of the design variable $\alpha$. The evaluation of $\mathrm{J}$ at the upper level is for the fixed value of $\alpha$ and $\beta=\beta^{*}$ and $\chi=\chi^{*}$. Thus, proper calculation of the gradient of $\mathrm{J}$ with respect to $\alpha$ must also include the effects of the changes in $\beta^{*}$ and $\chi^{*}$ with respect to changes in $\alpha$. This gradient of $\mathrm{J}$ with respect to $\alpha$ can of course be calculated by a direct finite difference method at the upper level. In this case, $\mathrm{J}$ is evaluated for small perturbations in $\alpha$, where the minimizations of $K$ and $L$ for $\beta^{*}$ and $\chi^{*}$ are repeated for each perturbation. The necessary gradient is then obtained by finite differencing of the perturbed values of $\mathrm{J}$.

Another means to calculate the gradient of $\mathrm{J}$ with respect to $\alpha$ is to use a chain rule approach. This results in a calculation for the gradient of $J$ at the upper level which directly involves terms which are the gradients of $\beta^{*}$ and $\chi^{*}$ with respect to $\alpha$. In this approach, the gradients of $\beta^{*}$ and $\chi^{*}$ with respect to $\alpha$ are calculated during the lower level minimizations of $K$ and $L$, and are passed to the upper level along with the optimized design variables $\beta^{*}$ and $\chi^{*}$ as illustrated in the figure. This can simplify the overall multilevel optimization algorithm because the lower level minimizations do not have to be repeated for every perturbation of $\alpha$ at the upper level. (Multiple solutions of the lower level optimization problems may still be required however, if the gradients of $\beta^{*}$ or $\chi^{*}$ with respect to $\alpha$ are calculated by a finite difference method, but they are independent of upper level perturbations of $\alpha$.)

In many cases, analytical expressions for the gradients of $\beta^{*}$ and $\chi^{*}$ with respect to $\alpha$ can be derived from the necessary conditions of optimality $13-15$ for the minimization of $K$ and $L$, also as shown in Figure 1. These expressions are evaluated once for $\beta=\beta^{*}$ and $\chi=$ $\chi^{*}$, and the gradient information is passed on to the upper level. Multiple solutions of the lower level optimization problems about a fixed value of the design variable $\alpha$ are eliminated by use of these analytical gradient expressions. 
A geometrical interpretation of the sensitivity of optimized solutions is shown in Figure 2a. In this figure, $M(u, p)$ represents a generic performance index to be minimized by selection of a design variable $u$, and $p$ is a parameter with some fixed nominal value for a solution of the optimization problem. A locus of optimal solutions as a function of the value of $p$ is shown in the figure labeled as $\mathbf{M}^{*}(\mathrm{p})$.

The sensitivity derivative of an optimal solution with respect to $p$ gives the slope and direction of the locus of optimized solutions at the parameter value corresponding to the optimal solution. This derivative has a component in both the $p$ and $u$ directions as shown in Figures 2.b and 2.c respectively. The projection of the sensitivity derivative onto the $M, p$ plane gives the change in the optimized performance index due to changes in the fixed value of the parameter $p$. The projection of the sensitivity derivative into the $M, u$ plane gives the change in the optimized performance index which is due to the change in the (optimal) $\mathbf{u}$. It would appear that this projection violates the necessary condition of the optimization problem since the derivative of $M$ with respect to arbitrary variations in u must be zero at the optimum. In other words, the derivative $\partial \mathrm{M} / \partial \mathrm{u}$ must be zero along a line parallel to the $u$ axis. This is in fact the case here also. The nonzero projection in the $M, u$ plane is caused by the constraint that the sensitivity derivative be calculated for $u$ $=\mathrm{u}^{*}(\mathrm{p})$. That is, the $\mathrm{M}, \mathrm{u}$ projection is really $\partial \mathrm{M} / \partial \mathrm{p}$ mapped from the $M, p$ plane by $u=u^{*}(p)$.

The sensitivity of the optimized performance index is an incomplete measure of the change in optimal solutions since there is no information on the change in the design variables due to parameter variations. This is particularly evident in the simple example of Figure 1, where the lower level minimization of $\mathrm{K}$ is considered. In that problem, the optimized performance index is $\mathrm{K}^{*}=0$ for all values of the parameter $\alpha$, but the optimizing design variable is $\beta^{*}=\alpha$. Use of the sensitivity of $K^{*}$ with respect to the parameter $\alpha$ would not yield any useable information for the upper level optimization, since that sensitivity is identically zero for all values of $\alpha$. In the example then, the square difference of $\beta$ from a desired value was used as the criteria at the upper level which is related to the lower level $\mathrm{K}$ minimization problem. The introduction and use of additional criteria to measure lower level performance at the upper levels is a requirement of multilevel optimization algorithms.

Several iterations of the conceptual problem of Figure 1 for the case $\bar{\beta}=\bar{\chi}=1$ are shown below as a final illustration of multilevel algorithms. Note in particular that once an $\alpha$ is selected, the lower level solutions for $\beta^{*}$ and $\chi^{*}$ are decoupled and are obtained independently of each other. (For the purposes of this illustration, a different scale factor in the computation for $\Delta \alpha$ was used at each step to limit the number of iterations.)

Iteration 1: $\left(\alpha_{1}=1\right.$ assumed to start $)$

$$
\begin{aligned}
& \alpha_{1}=1 \Rightarrow \beta_{1}^{*}=1 ; \chi_{1}^{*}=2 \\
& \mathrm{~J}_{1}=1 ; \frac{\mathrm{d}}{\mathrm{d} \alpha}=\alpha_{1}+\left(\beta_{1}^{*}-1\right)(1)+\left(\chi_{1}^{*}-1\right)(2)=3 \\
& \Delta \alpha_{1}=-\mathrm{J}_{1} \frac{\mathrm{dJ}^{-1}}{\mathrm{d \alpha}}=(-1) \frac{1}{3}=-\frac{1}{3}
\end{aligned}
$$

Iteration 2:

$$
\begin{aligned}
& \alpha_{2}=\alpha_{1}+\Delta \alpha_{1}=1-\frac{1}{3}=\frac{2}{3} \Rightarrow \beta_{2}^{*}=\frac{2}{3} ; \chi_{2}^{*}=\frac{4}{3} \\
& \mathrm{~J}_{2}=\frac{1}{3} ; \frac{\mathrm{d} J}{\mathrm{d \alpha}}=\alpha_{2}+\left(\beta_{2}^{*}-1\right)(1)+\left(\alpha_{2}^{*}-1\right)(2)=1 \\
& \Delta \alpha_{2}=-\frac{1}{2} \mathrm{~J}_{2} \frac{\mathrm{dJ}^{-1}}{\mathrm{d \alpha}}=-\frac{1}{6}(1)=-\frac{1}{6}
\end{aligned}
$$

Iteration 3:

$$
\begin{aligned}
& \alpha_{3}=\alpha_{2}+\Delta \alpha_{2}=\frac{2}{3} \cdot \frac{1}{6}=\frac{1}{2} \Rightarrow \beta_{3}^{*}=\frac{1}{2} ; \chi_{3}^{*}=1 \\
& \mathrm{~J}_{3}=\frac{1}{4} ; \frac{\mathrm{d}}{\mathrm{d \alpha}}=\alpha_{3}+\left(\beta_{3}^{*}-1\right)(1)+\left(\chi_{3}^{*}-1\right)(2)=0 \\
& \therefore \alpha_{3}=\alpha^{*}=\frac{1}{2}
\end{aligned}
$$

Analytical Solution: (Obtained by substituting the expressions for $\beta^{*}, \chi^{*}$, and their derivatives into the gradient of $\mathrm{J}$ with respect to $\alpha$ and setting the result equal to zero)

$$
\begin{aligned}
\frac{d}{d \alpha} & =\alpha+\left(\beta^{*}-\bar{\beta}\right) \frac{\partial \beta^{*}}{\partial \alpha}+\left(\chi^{*}-\bar{\chi}\right) \frac{\partial \chi^{*}}{\partial \alpha} \\
& =\alpha+(\alpha-\bar{\beta})(1)+(2 \alpha-\bar{\chi})(2)=6 \alpha-\bar{\beta}-\bar{\chi}=0 \\
\Rightarrow & \alpha^{*}=\frac{1}{6}(\bar{\beta}+2 \bar{\chi})=\frac{1}{6}(1+2)=\frac{1}{2}
\end{aligned}
$$




\section{Multilevel Structure/Control Law Design Approach}

A multilevel optimization based method can be developed for integrated structure/ control law design. Specific formulations for such algorithms are of course highly problem dependent, however, a general formulation which uses Linear Quadratic Gaussian optimal control law design methods to determine the control law is outlined below.

The general multilevel structure/control law design formulation is shown in Figure 3. In Figure 3, the structural design and the control law design are independent lower level design problems. These lower level designs are coordinated through the upper level optimization problem. The upper level optimization problem reflects the desired objectives of the integrated structure/control law design. For example, the upper level objective might be to reduce peak transient responses of the controlled system and to reduce the weight of the structure. The actual peak transient responses of the controlled system would come from analysis of the control law design at the lower level, while the actual structural weight would come from the lower level structural optimization. These could then be combined as a weighted sum of square errors between the actual and desired values to form a single upper level performance index. The upper level design variables would then be selected to minimize the objective function.

The values of the upper level design variables at any time are treated as fixed parameters for the lower level optimizations. These parameters define either the mathematical model of the structure or dynamic system to be controlled, or they define the performance index and/or constraints of the optimization problems, or both. The sensitivities of the optimized lower level solutions to these fixed parameters are computed and used in turn to compute the sensitivity of the related part of the upper level performance index. That is, these final sensitivities are the gradients necessary to complete the top level optimization. In the earlier example, one of the upper level design variables may be a local structural stiffness requirement, which appears as an equality constraint in the lower level structural optimization. The sensitivity of the structural weight to this parameter is computed at the lower level and returned for use in computing the part of the gradient of the upper level performance index which is related to structural weight. Another of the upper level design variables might be a mean square weight on control effort, which would appear directly in the performance index of the lower level LQG control law design problem. The sensitivity of the optimized LQG control law with respect to this parameter would then be used to compute the sensitivity of the peak transient response of the controlled system, as required to complete the upper level optimization.

For the purposes of this paper, the use of existing nonlinear programming based structural optimization and design sensitivity analysis methods is assumed. These methods may themselves be multilevel optimization algorithms, such as those of references 15 and 16 .

Also for this paper, the use of Linear Quadratic Gaussian optimal control law design methods is assumed. Expressions for the sensitivity of controlled system time, frequency, and stochastic responses in terms of state-space coefficient sensitivity matrices are available from the literature. The sensitivity of the optimized LQG control law to fixed parameters must be known to compute the necessary state-space coefficient sensitivity matrices. Analytical expressions for the sensitivity of the LQG gain matrices to fixed problem parameters have been derived elsewhere from the necessary conditions of optimality. ${ }^{17}$ The analytical expressions for the LQG sensitivity as well as analytical sensitivity equations for frequency responses, time responses, covariance responses, eigenvalues and singular values are summarized in reference 18.

\section{Two-Bar Truss Example}

Description - A two-bar truss structure/control law design problem was taken directly from the literature ${ }^{7}$ and used to validate both the analytical sensitivity analysis developments and the multilevel structure/control law design method. The nominal truss structure geometry, definitions, and second-order differential equations of motion are shown in Figure 4. Note that the bar cross sectional areas and the Young's Modulus are scaled in this example, although the natural frequencies of vibration are consistent with an actual structure of this size and concentrated mass. With these scalings, the material mass density $\left(=2.59 \times 10^{-6} \mathrm{lb}-\mathrm{sec}^{2} / \mathrm{in}^{4}\right)$ is such that the mass of the bars is negligible compared to the concentrated mass for the vibration equations of motion. The bar cross-sectional areas are, however, a direct measure of the actual truss structure weight, which is to be reduced as the structural design objective. 
State-space equations of motion for the truss are

$$
\begin{aligned}
&\left\{\begin{array}{l}
\dot{z}_{1} \\
\dot{z}_{2} \\
\dot{z}_{1} \\
z_{2}
\end{array}\right\}= {\left[\begin{array}{cc}
0 & I \\
-\frac{1}{m} K & -0.01 \frac{1}{m} K
\end{array}\right]\left\{\begin{array}{l}
z_{1} \\
z_{2} \\
z_{1} \\
z_{2}
\end{array}\right\} } \\
&+\left[\begin{array}{c}
0 \\
0 \\
\cos \theta \\
\sin \theta
\end{array}\right] F(t)+\left[\begin{array}{l}
0 \\
0 \\
0 \\
1
\end{array}\right] w(t) \\
&\left\{\begin{array}{l}
y_{1} \\
y_{2}
\end{array}\right\}=\left[\begin{array}{llll}
1 & 0 & 0 & 0 \\
0 & 1 & 0 & 0
\end{array}\right]\left\{\begin{array}{l}
z_{1} \\
z_{2} \\
\dot{z}_{1} \\
\dot{z}_{2}
\end{array}\right\}
\end{aligned}
$$

or

$$
\begin{aligned}
& \dot{x}=A x+B u+D w \\
& y=C x
\end{aligned}
$$

where the definitions of $x, y, u, w, A, B, C$, and D follow from the state equations, $I$ is a $2 \times 2$ identity matrix, and the stiffness matrix $\mathrm{K}$ is

$$
K=\frac{E}{51}\left[\begin{array}{cc}
A_{1}+A_{2} & 2\left(A_{1}-A_{2}\right) \\
2\left(A_{1}-A_{2}\right) & 4\left(A_{1}+A_{2}\right)
\end{array}\right]
$$

Note that structural damping of 0.01 has been assumed in this model.

An LQG optimal control law problem was formulated for the two-bar truss example. A cost function

$$
J=\lim _{\tau \rightarrow \infty} \frac{1}{\tau} E \int_{\delta}^{\tau}\left(y^{T} Q y+u^{T} R u\right) d \tau
$$

was assumed, where $E$ denotes expected value, and the weighting matrices $Q$ and $R$ are $Q=q I$ and $R=r$, where the nominal values of $q$ and $r$ are 1.0 and 0.01 , respectively. Noisy measured feedback signals were assumed as $\mathrm{z}=\mathrm{y}+\mathrm{v}$, where the noise $\mathrm{v}$ is a zero mean, Gaussian distributed, "white" noise with intensity matrix $\mathrm{V}=\mathrm{vl}$, with the nominal value of $\mathrm{v}=0.01$. The disturbance input w was assumed to be a zero mean,
Gaussian distributed, "white" noise as well, with intensity $\mathrm{W}=\mathrm{w}, \mathrm{w}=1.0$ nominally. The noises $w$ and $\mathrm{v}$ were assumed to be uncorrelated.

Four parameters were selected for use as design variables at the top level of the multilevel structure/control law design algorithm for the three design cases presented below. These are the scale factor $q\left(p_{1}\right)$, the scale factor $\mathbf{v}\left(\mathrm{p}_{2}\right)$, the cross-sectional area $A_{1}$ of truss bar $1\left(p_{3}\right)$, and the force application angle $\theta\left(\mathrm{p}_{4}\right)$. The upper level objective function for the three design cases was written to minimize the sum of the square difference between actual and desired design criteria as

$$
S=\sum_{i=1}^{n}\left(c_{a_{1}}-c_{d}\right)^{2}
$$

The design criteria for each design case are given below.

Design Case 1 - Design case one was formulated to improve closed-loop stability robustness. Seven measures of controlled system stability robustness were used in the top level objective function. These included increasing the damping of the two structural modes by moving their eigenvalues to the left in the complex plane, raising the minimum singular value of the return difference at three frequencies, and reducing the magnitude of the loop transfer function at two frequencies. These criteria are further described in Table 1.

The initial LQG optimal control law solution was computed for the nominal values of the design parameters. The initial values of the seven criteria and their sensitivity to each of the design variables were also computed. The sensitivity results for this initial iteration are summarized in Table 2. The sensitivity data was arranged accordingly in a gradient matrix and an incremental change in the four design variables was computed as

$$
\Delta p=\frac{\partial c^{\dagger}}{\partial p}\left(c_{d}-c_{a}\right)
$$

where $p$ is a vector of the four upper level design variables, $c$ is a vector of the seven design criteria, the subscripts $\mathrm{d}$ and a denote desired and actual values, and $\dagger$ denotes the matrix psuedo-inverse. New values of the four design variables were then selected as

$$
\mathrm{p}_{\mathrm{n}}=\mathrm{p}_{\mathrm{o}}+\frac{1}{2} \Delta \mathrm{p}
$$

where the subscripts $\mathrm{n}$ and o denote new and old and the factor of $1 / 2$ was selected to reduce the effects of the linearization error. This process was repeated for five iterations. 
The design results are shown in Figures 5 and 6 . In Figure 5, the design criteria iteration history is shown with the results normalized by the desired value, so that satisfaction of a criterion occurs for a value of 1.0. All seven criteria are moving toward satisfaction with each iteration. Figure 6 shows the history of the upper level design variables, normalized by their initial (starting) value. There is a large increase in the $\mathrm{V}$ matrix scale factor $v$, which is effectively tuning the Kalman Filter design, a slight increase in the $Q$ matrix scale factor $q$, which is tuning the regulator design, a slight increase in structural weight, and a decrease in the control force application angle. These results indicate that a combination of structure and control law design changes can be used to improve the overall stability robustness of a controlled system.

Design Case 2 - The second design case was formulated to tradeoff structure and control design objectives. The upper level design objective was written to improve six integrated design criteria. The criteria included the mean-square response and control energy due to a random disturbance input $w(t)$, the static controlled system structural deflections due to a static load, and the structural weight (directly related to the cross-sectional area of bar 1). The objective was formulated as in Case 1, with the desired values of the six criteria set to $95 \%$ of the initial values. The criteria are further described in Table 3 .

The normalized results are shown in the Figures 7 and 8. Both the control force and the structural weight in Figure 7 were reduced toward their desired values, while the other four criteria were actually increased. Looking at the upper level design variable iteration history in Figure 8 , the cross-sectional area was, reduced to $95 \%$ of the original value, reflecting the direct influence of it's magnitude in the upper level objective. The control force application angle was increased, the $\mathrm{Q}$ matrix scale factor $q$ was slightly decreased, and the $\mathrm{V}$ matrix scale factor $\mathrm{v}$ was increased $31 \%$. The conclusion here is that the actual magnitude of the cross-sectional area dominates the design.

Design Case 3 - Design Case 3 was the same as Design Case 2 except that the upper level design criteria were normalized by their initial values before the upper level objective function was formulated. The results for this case show that five of the six criteria are improved (Figure 9), the exception now being the structural weight which is increasing. The design parameter history in Figure 10 shows the $2 \%$ increase in bar cross-sectional area and also an 8-9\% increase in force application angle. The results show the dependence of the design on the magnitudes of the design criteria relative to one another. They also indicate that small increases in structural weight may be worthwhile in terms of controlled system performance. This is intuitively satisfying since decreases in structural deformations are often associated with increased structural weight.

\section{Conclusion}

A new approach to integrated structure/control law design of aeroelastic aircraft and large space structures has been presented. This approach uses multilevel optimization techniques which are are based on the theories of hierarchal problem decomposition, optimization, and sensitivity of optimum solutions. Unlike existing sequential or simultaneous methods, the structure and control law designs are obtained independently. Integration of the structure and control law designs is achieved through an optimization problem formulation in which the independent disciplinary designs are coordinated at an upper level. Multilevel optimization has been explained by use of a simple conceptual example, and a general multilevel structure/control law design algorithm outlined. Results for three integrated structure/control law designs of a two-bar truss example problem were presented. These results illustrate the multilevel design method and show the potential tradeoffs that are possible between the structure and control law designs. The examples point out once more the importance of the relative magnitudes of the design criteria to the final results.

\section{References}

[1] Junkins, J. L., and Rew, D. W., "Unified Optimization of Structures and Controllers", Large Space Structures: Dynamics and Control, Atluri, S. N. and Amos, A. K. editors, Springer-Verlag 1988.

[2] Haftka, R., Martinovic, Z., and Hallauer, W., "Enhanced Vibration Controllability by Minor Structural Modifications", AIAA Journal, Vol. 23, No. 8, August 1985, pg. 1260-1266.

[3] Salama, M., Hamidi, M., and Demsetz, L., "Optimization of Controlled Structures", JPL Proceedings of the Workshop on Identification and Control of Flexible Space Structures, 1984, pg. 311-327.

[4] Hale, A., "Integrated Structural/Control Synthesis via Set-Theoretic Methods", AIAA 85-0806, 26th Structures, Structural Dynamics, and Materials Conference, April 1985.

[5] Miller, D. F., Venkayya, V. B., and Tischler, V. A., "Integration of Structures and Controls - Some Computational Issues", $24^{\text {th }}$ Conference on Decision and Control, Ft. Lauderdale FL., Dec. 1985. 
[6] Messac, A., Turner, J., and Soosar, K., "An Integrated Control and Minimum Mass Structural Optimization Algorithm for Large Space Structures", JPL Proceedings of the Workshop on Identification and Control of Flexible Space Structures, 1984, pg. 231-266.

[7] Khot, N. S., Venkayya, V. B., Oz, H., Grandhi, R. V., and Eastep, F. E., "Optimal Structural Design with Control Gain Norm Constraint", AIAA 87$0019,25^{\text {th }}$ Aerospace Sciences Meeting, January 1987, Reno, NV.

[8] Sobieszczanski-Sobieski, J., "A Linear Decomposition Method for Large Optimization Problems - A Blueprint for Development", NASA TM-83248, Feb. 1982.

[9] Sobieszczanski-Sobieski, J., Barthelemy, J., and Giles, G., "Aerospace Engineering Design by Systematic Decomposition and Multilevel Optimization", ICAS-84-4.7.3, Sept. 1984.

[10] Mesarovic, M., Macko, D., and Takahara, Y., Theory of Hierarchical, Multilevel, Systems, Academic Press, 1970.

[11] Bernussou, J., and Titli, A., Interconnected Dynamical Systems: Stability, Decomposition, and Decentralization, North-Holland Publishing Co., 1982.

[12] Sobieszczanski-Sobieski, J., Barthelemy, J., and Riley, K., "Sensitivity of Optimum Solutions of Problem Parameters", AIAA Journal, Vol. 20, No. 9, Sept. 1982, pg. 1291-1299.
[13] Barthelemy, J., and Sobieszczanski-Sobieski, J., "Extrapolation of Optimum Design Based on Sensitivity Derivatives", AlAA Journal, Vol. 21, No. 5, May 1983, pg. 797-799.

[14] Barthelemy, J., and Sobieszczanski-Sobieski, J., "Optimum Sensitivity Derivatives of Objective Functions in Nonlinear Programming", AIAA Journal, Vol. 21, No. 6, June 1983, pg. 913-915.

[15] Sobieszczanski-Sobieski, J., James, B., and Dovi, A., "Structural Optimization by Multilevel Decomposition", AIAA 83-0832, 24th Structures, Structural Dynamics, and Materials Conference, May 1983.

[16] Sobieszczanski-Sobieski, J., James, B., and Riley, M., "Structural Optimization by Generalized, Multilevel Optimization", AIAA 85-0697, 26th Structures, Structural Dynamics, and Materials Conference, April 1985.

[17] Gilbert, M. G., "Design Parameter Sensitivity of Optimum LQG Control Laws for Application to an Integrated Interdisciplinary Design Methodology", PhD Dissertation, Purdue University, W. Lafayette IN, to be published, December 1989.

[18] Gilbert, M. G., "An Analytical Sensitivity Method For Use in Integrated Aeroservoelastic Aircraft Design", European Forum on Aeroelasticity and Structural Dynamics, Aachen, FRG, April 17-19, 1989. Also available as NASA TM 101583, May 1989.

Table 1. Case 1 Design Criteria

\begin{tabular}{cccl}
$\begin{array}{c}\text { Criteria } \\
\text { Number }\end{array}$ & $\begin{array}{c}\text { Original } \\
\text { Value }\end{array}$ & $\begin{array}{c}\text { Desired } \\
\text { Value }\end{array}$ & Description \\
\hline 1 & -0.506 & -0.531 & Real Parts of Closed Loop Structural Mode Pair 1 \\
2 & -1.452 & -1.524 & Real Parts of Closed Loop Structural Mode Pair 2 \\
3 & 0.834 & 0.900 & Return Difference Singular Value at $1.00 \mathrm{rad} / \mathrm{sec}$. \\
4 & 0.832 & 0.900 & Return Difference Singular Value at 3.35 rad/sec. \\
5 & 0.859 & 0.900 & Return Difference Singular Value at 5.99 rad/sec. \\
6 & 1.263 & 0.900 & Loop Transfer Magnitude at 5.21 rad/sec. \\
7 & 1.251 & 0.900 & Loop Transfer Magnitude at 5.37 rad/sec. \\
\hline
\end{tabular}


Table 2. Case 1 Original Design and Sensitivity Data for First Iteration

\begin{tabular}{|c|c|c|c|c|c|}
\hline $\begin{array}{l}\text { Criteria } \\
\text { Number }\end{array}$ & $\begin{array}{l}\text { Original } \\
\text { Value }\end{array}$ & $\frac{\partial \mathrm{c}_{i}}{\partial \mathrm{p}_{1}}$ & $\frac{\partial c_{1}}{\partial p_{2}}$ & $\frac{\partial c_{i}}{\partial p_{3}}$ & $\frac{\partial \mathrm{c}_{i}}{\partial \mathrm{p}_{4}}$ \\
\hline 1 & -0.506 & -0.210 & 0.000 & $-2.923 \times 10^{-4}$ & -0.762 \\
\hline 2 & -1.452 & -0.542 & 0.000 & $1.031 \times 10^{-3}$ & 0.851 \\
\hline 3 & 0.834 & $5.148 \times 10^{-3}$ & 4.274 & $2.874 \times 10^{-4}$ & $-3.891 \times 10^{-2}$ \\
\hline 4 & 0.832 & $-1.178 \times 10^{-2}$ & 4.441 & $3.652 \times 10^{-4}$ & $-3.922 \times 10^{-2}$ \\
\hline 5 & 0.859 & $-4.637 \times 10^{-2}$ & 1.962 & $1.416 \times 10^{-4}$ & $-8.860 \times 10^{-2}$ \\
\hline 6 & 1.263 & 0.451 & $-2.263 \times 10^{1}$ & $-4.245 \times 10^{-3}$ & 1.874 \\
\hline 7 & 1.251 & 0.461 & $-2.393 \times 10^{1}$ & $4.410 \times 10^{-3}$ & 1.820 \\
\hline
\end{tabular}

Table 3. Case 2 and Case 3 Design Criteria

\begin{tabular}{clll}
$\begin{array}{c}\text { Criteria } \\
\text { Number }\end{array}$ & $\begin{array}{c}\text { Original } \\
\text { Value }\end{array}$ & $\begin{array}{l}\text { Desired } \\
\text { Value }\end{array}$ & Description \\
\hline 1 & $1.194 \times 10^{-2}$ & $1.135 \times 10^{-2}$ & Mean-Square Deflection in x Direction \\
2 & $3.125 \times 10^{-2}$ & $2.968 \times 10^{-2}$ & Mean-Square Deflection in y Direction \\
3 & 0.646 & 0.614 & Mean-Square Control Force \\
4 & $3.629 \times 10^{-2}$ & $3.447 \times 10^{-2}$ & Steady-State $\times$ Deflection to Step Load \\
5 & $4.490 \times 10^{-2}$ & $4.266 \times 10^{-2}$ & Steady-State y Deflection to Step Load \\
6 & $5.000 \times 10^{2}$ & $4.750 \times 10^{2}$ & Bar 1 Cross-Sectional Area (Truss Weight) \\
\hline \hline
\end{tabular}

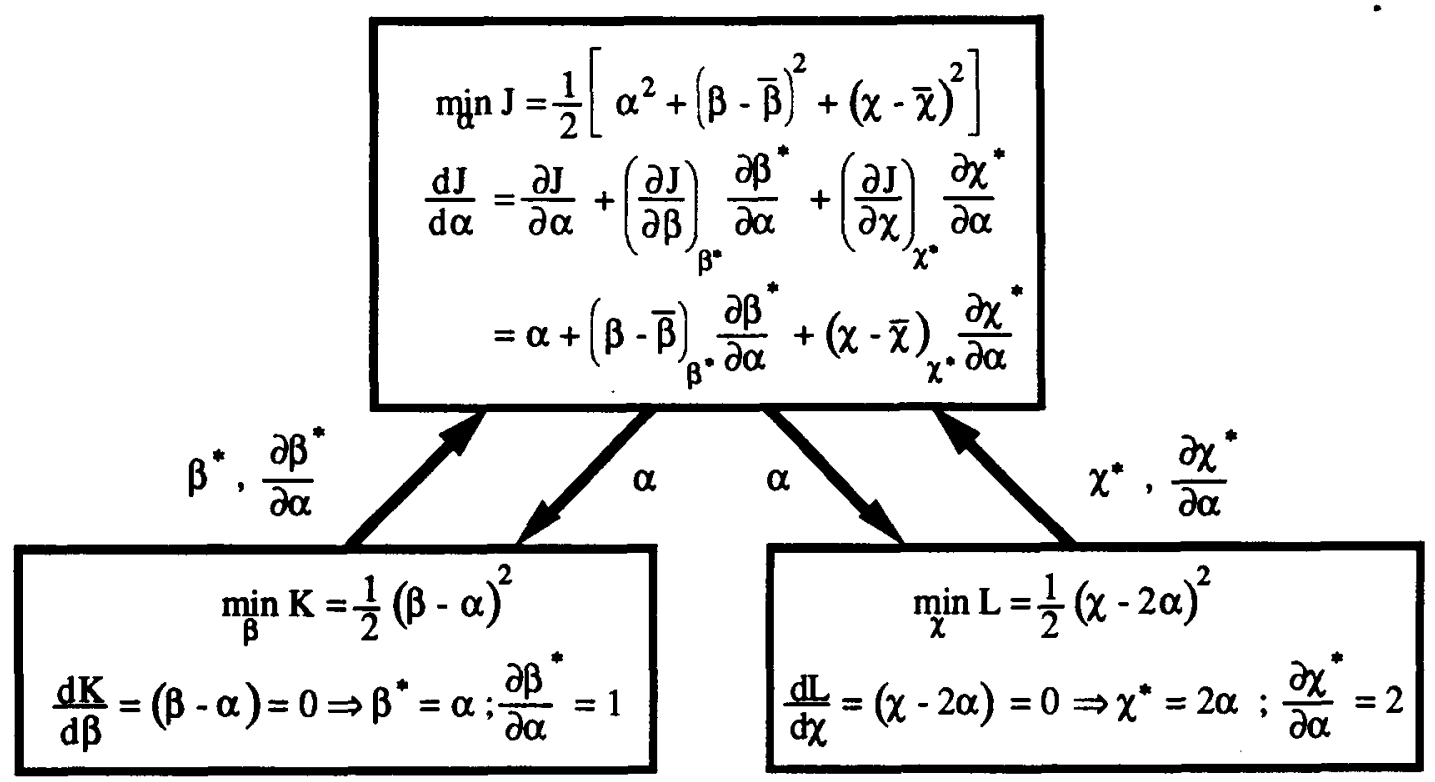

Figure 1 - Conceptual Multilevel Optimization Problem 


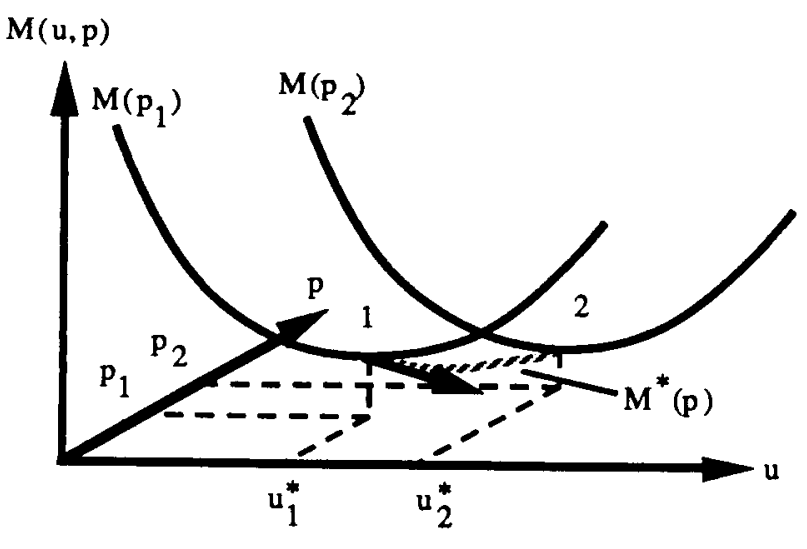

Figure 2a - Geometrical Interpretation of Sensitivity of Optimum Concept

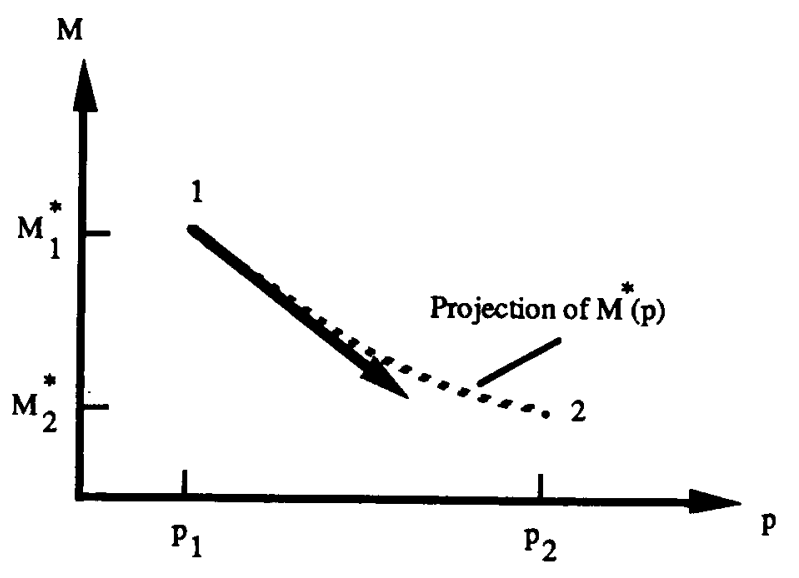

Figure $2 \mathrm{~b}$ - M,p Plane Projection

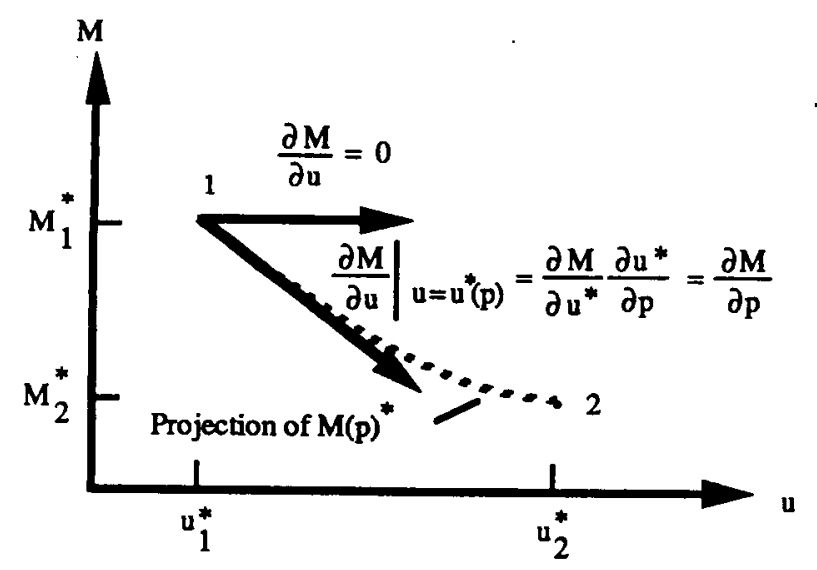

Figure 2c - M,u Plane Projection

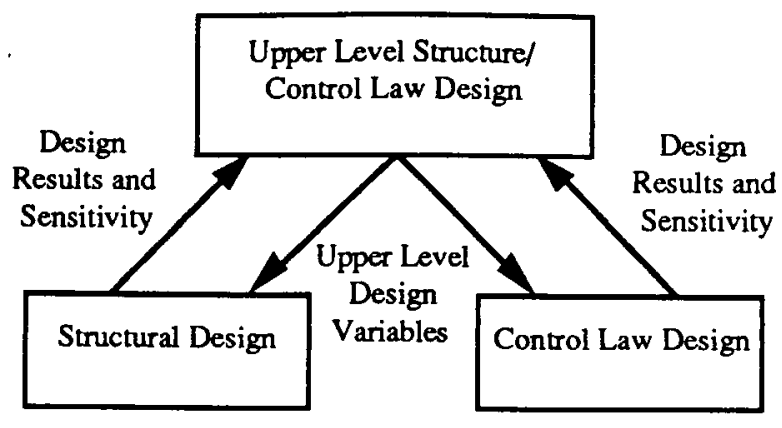

Figure 3 - General Multilevel Structure/Control Law Design Algorithm

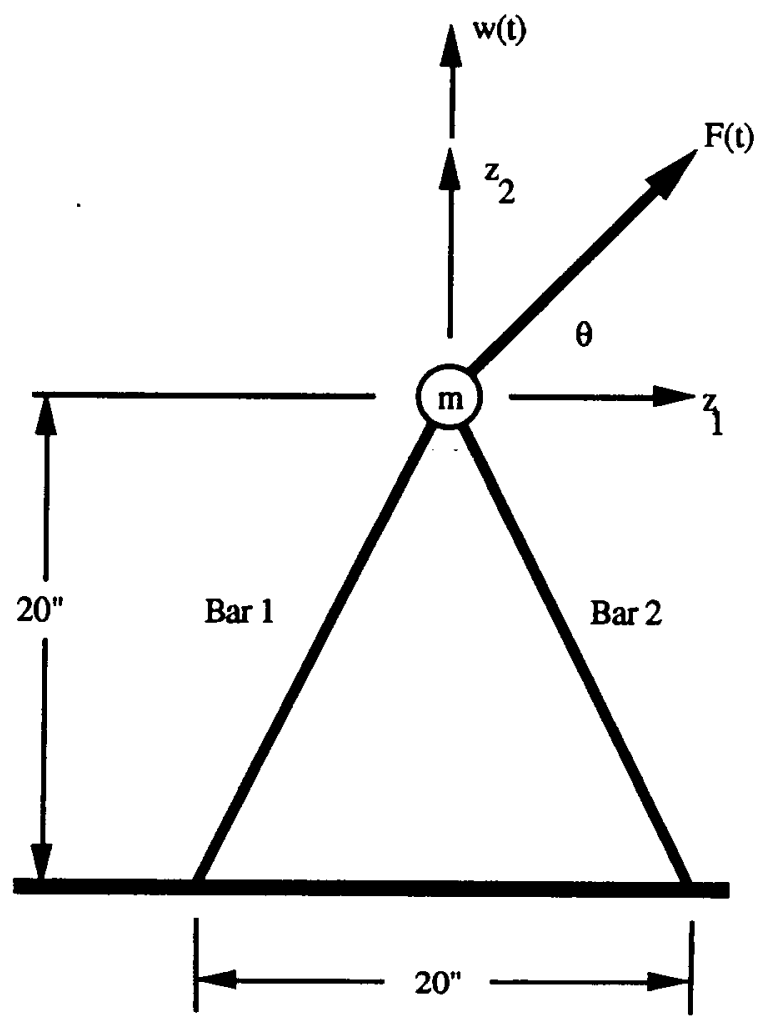

Mass $=\mathrm{m}=2$

Area $\operatorname{Bar} 1=A_{1}=500$

Area $\operatorname{Bar} 2=A_{2}=1000$

Length Bar $1=$ Length Bar $2=1=22.36 "$

Force Application Angle $=\theta=45^{\circ}$

Young's Modulus E $=1$

Figure 4 - Two-Bar Truss Geometry and Data 


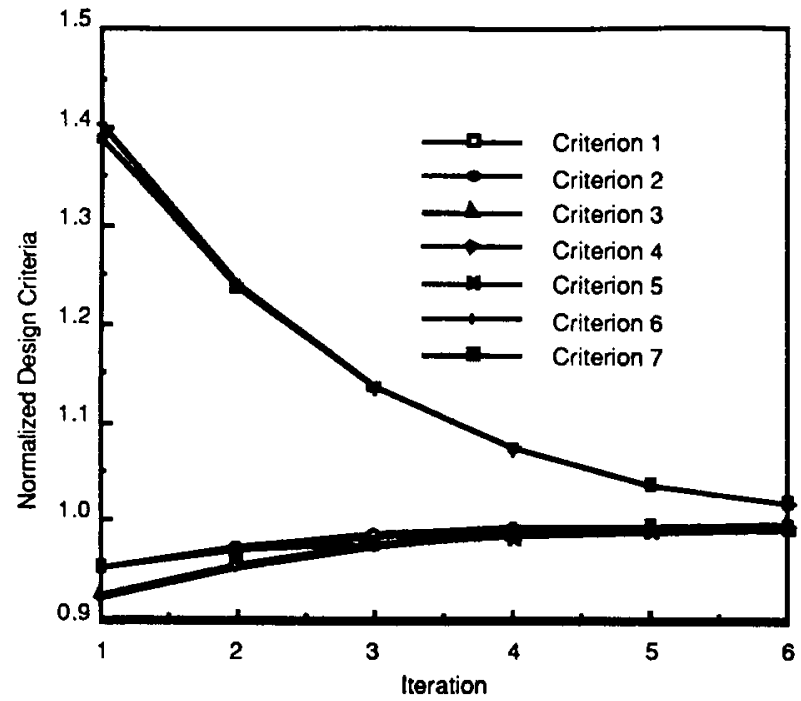

Figure 5 - Case 1 Design Criteria Iteration History

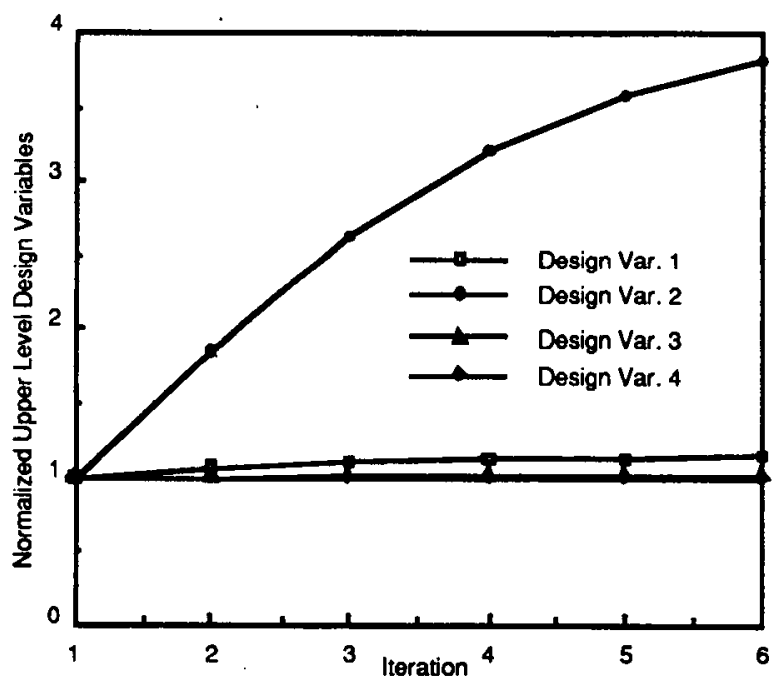

Figure 6 - Case 1 Upper Level Design Variable History

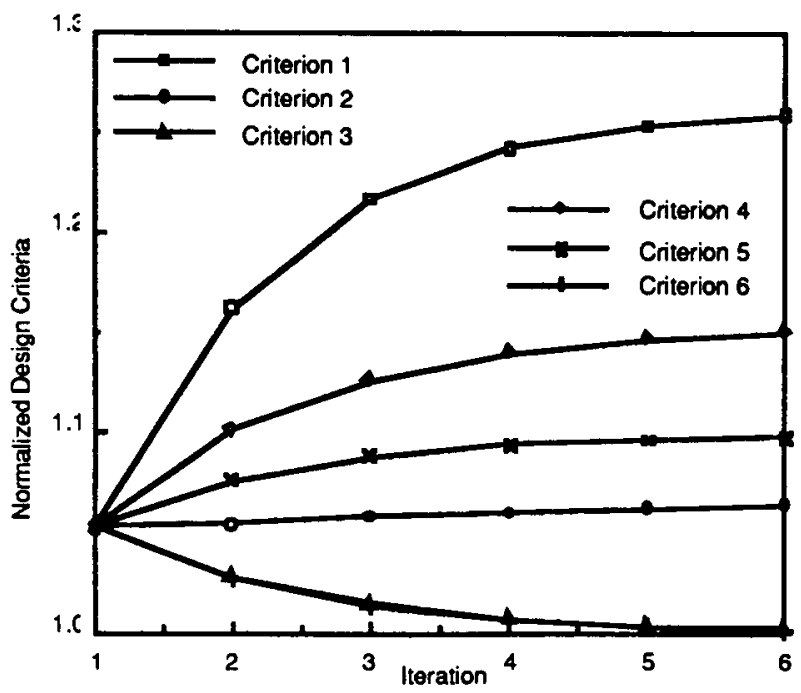

Figure 7 - Case 2 Design Criteria Iteration History

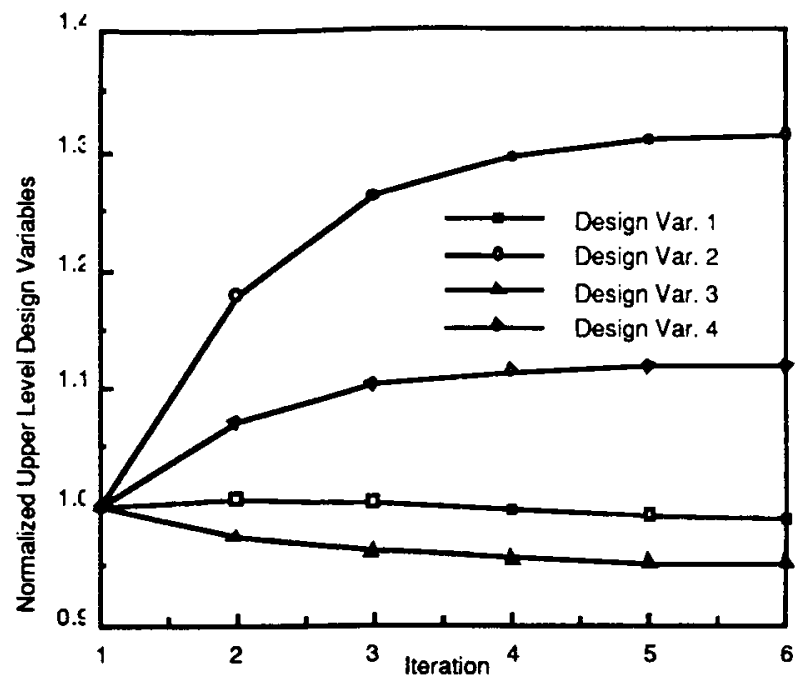

Figure 8 - Case 2 Upper Level Design Variable History

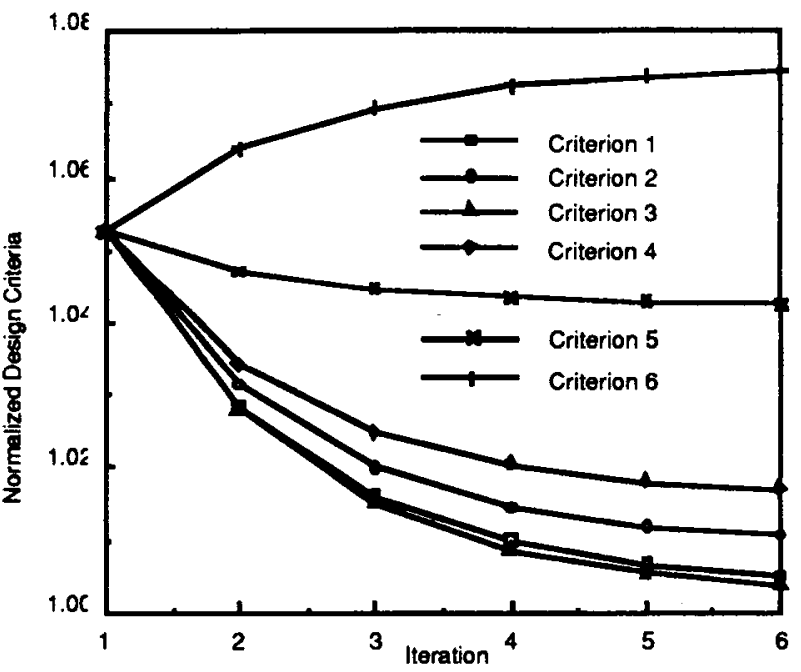

Figure 9 - Case 3 Design Criteria Iteration History

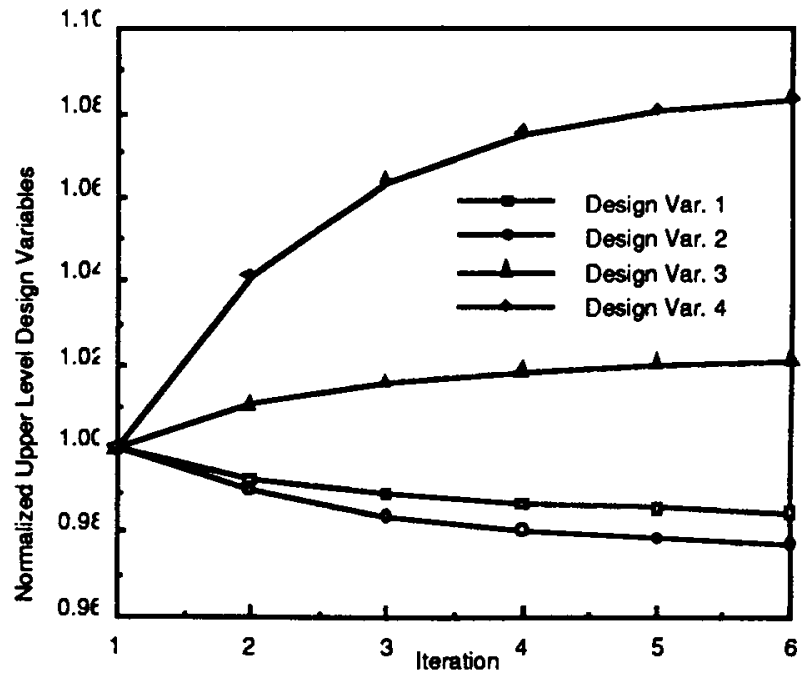

Figure 10 - Case 3 Upper Level Design Variable History 


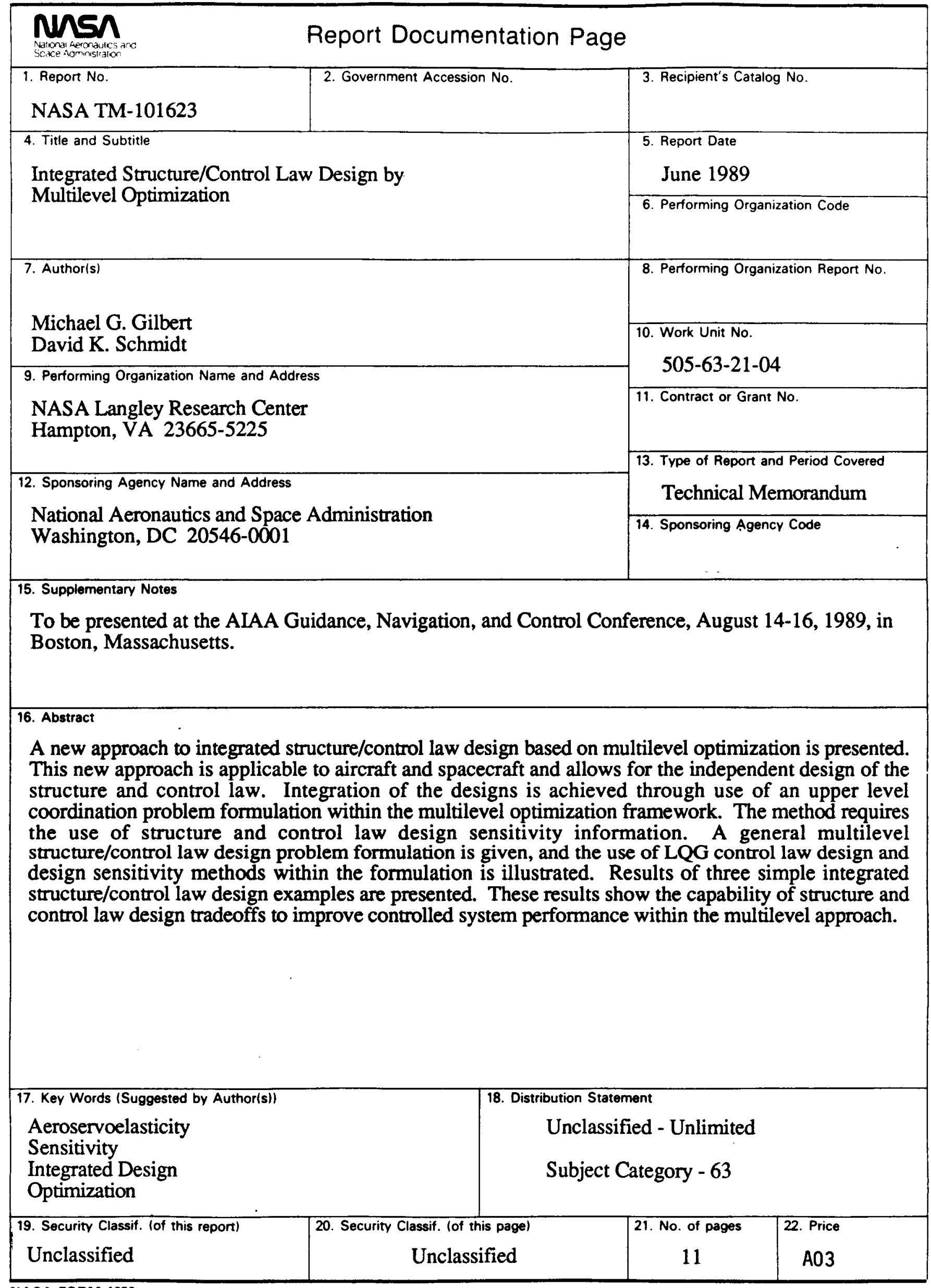

